Bazyli Degórski O.S.P.P.E. ${ }^{1}$

\title{
Manoscritti inclassificabili della Vita S. Pauli Monachi Thebaei di san Girolamo
}

Analizziamo tutti i codici manoscritti della Vita S. Pauli Monachi Thebaei di san Girolamo che sono ancora inclassificabili, ossia che non fanno parte di alcuna famiglia della tradizione manoscritta di quest'opera geronimiana. Le prove dell'eventuale parentela di un manoscritto inclassificabile con un gruppo sono presentate nel seguente ordine: all'inizio vi è un elenco dei manoscritti inclassificabili, con il numero, il nome, la data e la provenienza di ciascuno. Poi, seguono le descrizioni dei manoscritti. Infine, vengono fornite le prove sulle quali si basa la classificazione, e ciò di solito nel seguente ordine: varianti comuni a tutti i gruppi, che stabiliscono la loro unità come famiglia; qualsiasi variante che prova che un manoscritto è la copia di un altro; le varianti comuni ai diversi componenti del gruppo che mostrano i sotto-raggruppamenti e le parentele immediate dei vari codici. Tutte le aggiunte sono chiuse in parentesi a punta: $<>$, mentre le lacune vengono indicate con tre trattini brevi: ---.

\section{Descrizione dei codici ancora inclassificabili ${ }^{2}$}

Forniamo ora l'elenco di tutti i codici manoscritti della Vita Pauli ancora inclassificabili, ossia che non fanno parte di alcuna famiglia della tradizione manoscritta dell'opera geronimiana.

1 Prof. dr. hab. Bazyli Degórski O.S.P.P.E., professore di patrologia e di teologia dogmatica presso la Facoltà di Teologia della Pontificia Università S. Tommaso d'Aquino a Roma, e professore di patrologia e di antropologia patristica presso la Pontificia Facoltà Teologica "Teresianum" a Roma, e-mail: osppe.roma@gmail.com, ORCID: 0000-0003-1997-2769.

2 Cf. J.F. Cherf, The Latin Manuscript Tradition of the "Vita Sancti Pauli", in: Studies in the Text Tradition of. St. Jerome's "Vitae Patrum”, red. W.A. Oldfather, Urbana 1943, 133-142; B. Degórski, Edizione critica della "Vita Sancti Pauli Primi Eremitae” di San Girolamo, diss. Institutum Patristicum Augustinianum, I-VI, Roma 1987 (dattiloscritto), 1500-1506, 1511, 1513-1514, 1516; B. Degórski, Edizione critica della "Vita Sancti Pauli Primi Eremitae” di Girolamo, Roma 1987, 58a-58b. 


\begin{tabular}{|c|c|c|c|}
\hline $\mathbf{N}^{\circ}$ codice & Nome del codice & Secolo & Provenienza \\
\hline 3. & Bernensis Latinus 111 & $\mathrm{XII} / \mathrm{XIII}$ & sconosciuta \\
\hline 5. & Bruxellensis 64 & XIII & Würzburg (S. Kilian) \\
\hline 7. & Bruxellensis $4815 / 18$ & XII & Villers-la-Ville \\
\hline 13. & Cameracensis 864 (767) & XI & Cambrai \\
\hline 19. & Dresdensis A 62 cod. D & $\mathrm{X}$ & Brauweiler \\
\hline 23. & Laurentianus pl. 19, cod. 16 & $\mathrm{XI} / \mathrm{XII}$ & sconosciuta \\
\hline $23 \mathrm{a}$. & Iagellonicus Cracoviensis 2265 AA XII 9 & $\mathrm{XV} / \mathrm{XVI}$ & Cracovia (S. Egidio) \\
\hline 26. & Matritensis 10007 & a. 902 & Toledo \\
\hline 45. & Parisinus Latinus 3784 & $\mathrm{X} / \mathrm{XI}$ & Limoges \\
\hline 46. & Parisinus Latinus 5314 & $\mathrm{XI} / \mathrm{XII}$ & Limoges \\
\hline 48. & Parisinus Latinus 5385 & $X$ & Ferrières \\
\hline 49. & Parisinus Latinus 9741 & $\mathrm{XII} / \mathrm{XIII}$ & Trier \\
\hline 63. & Casanatensis Codex 718 (B. I . 3) & XI & sconosciuta \\
\hline 66. & Lateranensis Codex A. 79 & $\mathrm{XI} / \mathrm{XII}$ & Roma (?) \\
\hline 67. & Farfensis 5 (270) & XIII & Gaeta \\
\hline 69. & Sessorianus 5 (XXIX) & $\mathrm{XI}$ & Nonantola (?) \\
\hline 73. & Vallicellanus Tomus III & $\mathrm{XII} / \mathrm{XIII}$ & sconosciuta \\
\hline 92. & Vaticanus Latinus 600 (153) & XIV & Roma (?) \\
\hline 103. & Vaticanus Latinus 5257 & $\mathrm{XV}$ & Verona (?) \\
\hline 104. & Vaticanus Latinus 5411 & $\mathrm{XI} / \mathrm{XII}$ & sconosciuta \\
\hline 108. & Vaticanus Latinus 7014 & XII & sconosciuta \\
\hline 109. & Vaticanus Latinus 9309 & XIII & sconosciuta \\
\hline 110. & Vaticanus Latinus 13393 & IX & sconosciuta \\
\hline 111. & Vaticanus Barberinianus Latinus 469 & XV & sconosciuta \\
\hline 112. & Vaticanus Barberinianus Latinus 586 & $\mathrm{XI} / \mathrm{XII}$ & sconosciuta \\
\hline 132. & Vaticanus Urbinas Latinus 396 (743) & $\mathrm{XV}$ & sconosciuta \\
\hline 134. & Basilicanus A. 2 & $\mathrm{X} / \mathrm{XI}$ & sconosciuta \\
\hline 135. & Basilicanus A. 4 & XI & sconosciuta \\
\hline 139. & Vindobonensis Latinus 420 & XI & Salisburgo \\
\hline 141. & $\begin{array}{c}\text { Vindobonensis S. Mariae ad Scotos } 409 \\
(324)\end{array}$ & XV & Vienna \\
\hline
\end{tabular}


I Bernensis Lat. 111, Bruxellensis $64^{3}$, Cameracensis $864(767)^{4}$, Dresdensis A 62 cod. $\mathrm{D}^{5}$, Laurentianus pl. 19, cod. $16^{6}$, Matritensis $10007^{7}$, Parisinus Lat. $3784^{8}$, Parisinus Lat. 53149, Parisinus Lat. $5385^{10}$, Parisinus Lat. 9741,

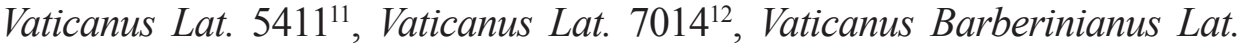

3 A proposito del Bruxellensis 64, cf. G. Philippart, Le légendier du Schottenkloster de Wurtzbourg (Cod. Bruxellensis 64), AnBol 89 (1971) 370.

4 A proposito del Cameracensis 864 (767), cf. E.M. Morales, Edición crítica de "De monacho captivo" ("Vita Malchi”) de San Jerónimo, diss. Institutum Patristicum Augustinianum (dattiloscritto), Roma 1991, passim.

A proposito del Dresdensis A 62 cod. D., cf. Morales, Edición crítica, passim.

6 A proposito del Laurentianus pl. 19, cod. 16, cf. Morales, Edición crítica, passim.

7 A proposito del Matritensis 10007, cf. Morales, Edición crítica, passim.

8 A proposito del Parisinus Lat. 3784, cf. Morales, Edición crítica, passim.

9 A proposito del Parisinus Lat. 5314, cf. Morales, Edición crítica, passim.

10 A proposito del Parisinus Lat. 5385, cf. Morales, Edición crítica, passim.

11 A proposito del Vaticanus Lat. 5411, cf. F. Dolbeau - P.-Y. Lambert - C. Lévy - P. Petitmengin - J.-Y. Tilliette, Les Vies latines de sainte Pélagie. Inventaire des textes publiés et inédits, RechAug 12 (1977) 300; F. Dolbeau - C. Lévy - P. Petitmengin - J.-P. Rothschild J.-Y. Tilliette, Les Vies latines de Sainte Pélagie. II. Compléments à l'nventaire et classement des manuscrits du texte B, RechAug 15 (1980) 290; B. Degórski, La “conlatio codicum” della "Vita S. Pauli Primi Eremitae”" (dall'inizio del primo capitolo sino alle parole: "[ ...] incitata sunt studia” dello stesso capitolo), DPa 1 (1988) 57-101; B. Degórski, La "conlatio codicum” della "Vita S. Pauli Primi Eremitae" di Girolamo, dall'inizio del II capitolo sino alle parole: “[...] pro Christi nomine gladio percuti” dello stesso capitolo, VoxP 9 (1989) 839-866; B. Degórski, La "conlatio codicum" della "Vita Sancti Pauli Primi Eremitae" di San Girolamo (dalle parole: "Amathas vero et Macarius" del primo capitolo sino alla fine dello stesso capitolo), RT 37 (1990) fasc. 4, 119-164; B. Degórski, La "conlatio codicum” della "Vita S. Pauli Primi Eremitae” di Girolamo, dall'inizio del II capitolo sino alle parole: "[ ...] pro Christi nomine gladio percuti” dello stesso capitolo, VoxP 9 (1989) 839-866; B. Degórski, La "conlatio [ ...] dalle parole: "Verum hostis [ ...], cit., 251-271; Morales, Edición crítica, pp. 128, 177-178 e passim; B. Degórski, I manoscritti delle biblioteche di Roma contenenti le traduzioni latine della Vita S. Antonii di sant'Atanasio. Alle origini della letteratura monastica, in: Cristianesimo Latino e cultura Greca sino al sec. IV. XXI Incontro di studiosi dell'antichità cristiana. Roma, 7-9 maggio 1992, Roma 1993 = SEA 42 (1993) 290.

12 A proposito del Vaticanus Lat. 7014, cf. P. Paschini, Ricerche agiografiche, RivAC 2 (1925) 181, 190; J.E. Cross - C.J. Tuplin, An Unrecorded Variant of the "Passio S. Christinae" and the "Old English Martyrology", "Traditio" 36 (1980) 208; G.N. Verrando, Liberio-Felice. Osservazioni e rettifiche di carattere storico-agiografico, "Rivista di storia della Chiesa in Italia" 35 (1981) 125. 
$586^{13}$, Basilicanus A. $2^{14}$, Vindobonensis Lat. 420 nonché Vindobonensis $S$.

13 A proposito del Vaticanus Barberinianus Lat. 586, cf. L. Bethmann, Nachrichten über die von ihm für die "Monumenta Germaniae historica" benutzten Sammlungen von Handschriften und Urkunden Italiens, aus dem Jahre 1854, "Archiv der Gesellschaft für ältere deutsche Geschichtskunde” 12 (1872) 380; Paschini, Ricerche, pp. 172-173, 181, 186, 191; G. Mercati, Opere minori raccolte in occasione del settantesimo natalizio sotto gli auspicii di S. S. Pio XI, volume I (1891-1897), Città del Vaticano 1937 = ST 76 (1937) 233; A. Lentini, Il sermone di S. Bertario su S. Scolastica, "Benedictina” 1 (1947) 202, 204, 206, 211-231; F. de Meeûs, Études grégoriennes, "Scriptorium” 10 (1956) 281; B. Baroffio, S. Gaudenzio di Novara e S. Gaudenzio di Fiesole, "Novarien” 2 (1968) 223; R.B.C. Huygens, Deux sermonnaires médiévaux: Tétère de Nevers et Giraud de Barri. Textes inédits, SM 10 (1969) 271; Z. Zafarana, Indici dei volumi I-VI, "Studi gregoriani per la storia della «libertas Ecclesiae»" 8 (1970) 194; Philippart, Le légendier, p. 370; E.G. Garrison, A Third "S. Bononio Manuscript" for S. Michele a Marturi, "Bibliofilia" 78 (1976) 99; D.N. Kissane, Uita Metrica Sanctae Brigidae: a critical edition with introduction, commentary and indexes, "Proceedings of the Royal Irish Academy" 77 (1977) 59, 73, 76-143, 156-179; W. Weismann, Die "Passio Genesii mimi” (BHL 3320), "Mittellateinisches Jahrbuch" 12 (1977) 31-43; P. Conte, Osservazioni sulla leggenda di S. Cerbonio, vescovo di Populonia (H 575), "Aevum” 52 (1978) 236; A.M. Orselli, La città altomedievale e il suo santo patrono: (ancora una volta) il "campione", pavese, "Rivista di Storia della Chiesa in Italia" 32 (1978) 69; U. Zanetti, Les Passions des SS. Nazaire, Gervais, Protais et Celse, AnBol 97 (1979) 77; Cross - Tuplin, An Unrecorded Variant, pp. 208-209, 219; F. Dolbeau, Un plagiat anonyme de la "Vita S. Columbani", “Archivum Bobiense" 3 (1981) 60-63; N. Verrando, Liberio-Felice, p. 125; N. Verrando, La "Passio Callisti” e il santuario della Via Aurelia, "Mélanges de l'École Française de Rome. Antiquité" 96 (1984) 1053, 1074-1075; Lentini, Il sermone di S. Bertario, pp. 373, 384-407; O. Limone, La tradizione manoscritta della "Vita Gregorii Magni” di Paolo Diacono (B.H.L. 3639). Censimento dei testimoni, SM 29 (1988) 911-948; P. Tomea, L'agiografia milanese nei secoli XI e XII. Linee di tendenza e problemi. I, in: Atti dell'111 Congresso internazionale di studi sull'Alto Medioevo, Milano 26-30 ottobre 1987, Spoleto 1989, 685; Degórski, La “conlatio [ ...] sino alle parole: " [ ...] incitata sunt, pp. 57-101; Degórski, La "conlatio [ ...] sino alle parole: “" ...] pro Christi nomine, pp. 839-866; Degórski, La "conlatio [ ...] dalle parole: “Amathas, pp. 119-164; Degórski, La "conlatio [ ...] gladio percuti” dello stesso capitolo, pp. 839-866; Degórski, La “conlatio [ ...] dalle parole: "Verum hostis, pp. 251-271; Degórski, I manoscritti delle biblioteche di Roma, pp. 292-293.

14 A proposito del Basilicanus A. 2, cf. G.B. De Rossi, I santi Quattro Coronati e la loro chiesa sul Celio, "Bollettino di archeologia cristiana" 3/4 (1879) 74; A. Wilmart, Une version latine inédite de la vie de saint Antoine, RBen 31 (1914-1919) 164-173; A. Wilmart, Les rédactions latines de la vie d'Abraham ermite, RBen 50 (1938) 225-239; F. De Marco, Censimento dei codici dei secoli X-XII. Roma: Biblioteca Apostolica Vaticana, Archivio di S. Pietro, SM 11 (1970) 1103-1104; G. Bartelink, Observations de critique textuelle sur la plus 
Mariae ad Scotos 409 (324) furono descritti da Cherf, e le nostre indagini ne attibuirono soltanto delle piccole precisazioni ed aggiunte ${ }^{15}$. Qui sotto, quindi, forniamo la descrizione di tutti i codici ancora inclassificabili della Vita che non furono esaminati da Cherf $^{16}$ :

23 a. Iagellonicus Cracoviensis 2265 AA XII $9^{17}$, di carta (in $4^{\circ}$ ), conservato nella Biblioteca dell'Università Iagellonica di Cracovia, XV/XVI s., 630 pagine scritte e due vuote. La Vita comincia a p. 461: Inter multos, e finisce a p. 470: [ ...] cum regnis suis. Il codice fu scritto da diverse mani. La parte nella quale si trova la Vita, fu copiata da un certo Jakubek [= Giacomino] da Lublino. Infatti, così leggiamo a p. 454: Verba sunt $b$. Hieronimi De monachis, vt sequuntur. [...]. Amen, Jacubek z Lublyna. Il codice proviene, però, da Cracovia. Infatti, almeno una parte di esso apparteneva alla chiesa di sant'Egidio di Cracovia, dato che alle pagine 480-481 leggiamo: Ecclesia collegiata sci $i$ Egidij castro Cracoviensi. In seguito, da partire dal 1536, passò al collegio dei teologi di Cracovia, perché in quell'anno fu rilegato proprio per quel collegio. Ciò avvenne grazie a Pietro Tomicki (Tomizio), vescovo di Cracovia: 1536,

ancienne version latine de la Vie de saint Antoine par saint Athanase, RBen 81 (1971) 92-95; F. Halkin, Sainte Tatiana: légende grecque d'une "martyre romaine”, AnBol 89 (1971) 267; M. Murjanov, “Tatjanin den"” nella cultura russa, OCP 38 (1972) 249; S.G. Axters, Na honderd jaar vroomheidsgeschiedenis, Gent 1973, 234; L.W. Barnard, The date of St. Athanasius' Vita Antonii, VigCh 28 (1974) 169; P. Supino Martini, Carolina romana e minuscola romanesca. Appunti per una storia della scrittura latina in Roma tra IX e XII secolo, SM 15 (1974) 781, 791; F. Dolbeau, Notes sur deux collections hagiographiques conservées à la Bibliothèque Vaticane, "Mélanges de l'École Française de Rome. Moyen âge - temps modernes" 87 (1975) 416; G.J.M. Bartelink, Grécismes lexicologiques et syntaxiques dans les traductions latines du IVe siècle de la "Vita Antonii" d'Athanase, "Mnemosyne" 30 (1977) 388; F. Dolbeau, Le légendier de la cathédrale de Bovino, AnBol 96 (1978) 125, 130; P. Supino Martini - A. Petrucci, Materiali ed ipotesi per una storia della cultura scritta nella Roma del IX secolo, "Scrittura" 2 (1978) 55; V. Tandoi, Sul testo della "Vita Antonii" di Atanasio, nella più antica versione latina (note marginali a un'edizione critica recente), "Studi italiani di filologia classica" 50 (1978) 161; G.J.M. Bartelink, Die älteste lateinische Übersetzung der "Vita Antonii" des Athanasius im Lichte der Lesarten einiger griechischer Handschriften, "Revue d'histoire des textes" 11 (1981) 397-413; M.G. Bianco, Gli intenti del traduttore anonimo della "Vita Antonii" di Atanasio e le sue doti letterarie, "Studi storico religiosi" 5 (1981) 223; I. Scicolone, Il cardinale Giuseppe Tomasi di Lampedusa e gli inizi della scienza liturgica, "Regnum Dei" 38 (1982) 51; Degórski, I manoscritti delle biblioteche di Roma, pp. 272-273.

15 Cf. Cherf, The Latin Manuscript, pp. 133-142; Degórski, Edizione critica [ ...] di San Girolamo, pp. 1500-1506, 1511, 1513-1514, 1516.

16 Cf. Degórski, Edizione critica [ ...] di San Girolamo, pp. 1512-1515.

17 A proposito di questo codice cf. B. Degórski, Un tardivo manoscritto di Cracovia contenente la "Vita Sancti Pauli Primi Eremitae” di San Girolamo, VoxP 8 (1988) 429-434. 
legatus pro Collegio theologorum per $R$ [everendissimum $] D[$ ominum $]$ Petrum Thomicium, epum Cracoviensem. Il testo della Vita fu copiato con una scrittura gotica corsiva, abbastanza difficile da leggere. Le correzioni sono poche e fatte da una seconda mano contemporanea allo scrivano.

63. Casanatensis Codex 718 (B.I.3) $^{18}$, XI s., 52 x $36 \mathrm{~cm}, 232$ fogli a due colonne con 50 righe di testo e circa 29 lettere in ogni riga. La Vita comincia al foglio 70 v: IIII. Idus IANUARII. INCIP[IT] UITA Ś PAULI HER>., e finisce al foglio $72 \mathrm{v}$ : Vnde grās agam'oms omipotenti dō, qui tanta mirabilia coruscare dignatus $\hat{e}$ in scĩs suis, ut ipso adiuuante mereamus eor[um] saluari precibus. AMEN. Il codice si trova nella "Biblioteca Casanatense" a Roma. La provenienza è sconosciuta. La scrittura è comune, scritta molto bene. Abbraviazioni comuni. Nessuna correzione.

66. Lateranensis Codex A. $79^{19}$, XI o XII s., 52 x $37 \mathrm{~cm}, 281$ fogli a due colonne. La Vita comincia al foglio $34 \mathrm{v}$ : Incip uita uel actus sci $i$ pauli ${ }^{20} * * * * *$ primi heremite, edita a sco ieronimo prb'o beth- (al foglio 35:) leem, e finisce al foglio 37: Finit uita beati pauli monachi, quā scripsit hieronimus ${ }^{21}$ pbrrrt [sic!], qui fuit ante sĉm antoniū quē ipse sepeliuit in heremo. Hic nūquā fuit in ciuitate $p$ 'quā in solitudine moratus est $t^{22}$. Il codice è conservato nell' "Archivio del Capitolo di San Giovanni in Laterano" a Roma, dove probabilmente fu scritto. Scrittura comune. Tantissime correzioni e abrasioni. Abbreviazioni frequenti, ma ordinarie.

67. Farfensis $5(270)^{23}$, XIII s., $238 \times 178$ mm., 426 pp. con ca. 53 lettere in ogni riga. La Vita comincia a p. 82: Incipit uita pauli primi heremite descripta a beato ieronimo ${ }^{24}$, e termina a p. 89: Explicit uita pauli primi hèmite. Il codice si trova nella "Biblioteca Nazionale Centrale Vittorio Emanuele" a Roma. Proviene dalla Biblioteca dell'Abbazia di Farfa, ma

18 A proposito di questo codice cf. Degórski, I manoscritti delle biblioteche di Roma, p. 277.

19 A proposito di questo codice, cf. Degórski, I manoscritti delle biblioteche di Roma, p. 276.

20 "Pauli" prima manus delevit.

21 Littera " $h$ ", in verbo "hieronimus", tertia manus super lineam addidit.

22 Omnia secunda manu super rasuram.

23 A proposito di questo codice, cf. Morales, Edición crítica, passim; Degórski, I manoscritti delle biblioteche di Roma, pp. 278-279.

24 Verba "Incipit uita pauli primi heremite descripta a" secunda manu, ut videtur, super rasuram scripta sunt". 
"erat prius Monasterii Sancti Angeli de Caieta"25. La scrittura è una gotica. Abbreviazioni numerose. Poche correzioni.

69. Sessorianus 5 (XXIX), XI s., 532 x $362 \mathrm{~mm}$., 248 fogli a due colonne, e ca. 36 lettere in ogni riga. La Vita comincia al foglio 52: IIII. ID' IAN UITA SPPP HEREMITE, e termina al foglio 54: EXPLICIT UITA BEATI PAULI MONACHI QUI FUIT ANTE SCM. ANTONIUM. QUE IPSE SEPELIUIT IN HEREMO. HIC NUMQUÄ FUIT IN CIUITATIB; POSTQU'A IN SOLITUDINE DEMORATUS EST. Il codice si trova nella "Biblioteca Nazionale Centrale Vittorio Emanuele" a Roma. A proposito della sua provenienza, così scrive Poncelet: "Fuisse prius codicem abbatiae Nonantulanae asseritur in catalogo manuscripto codicum bibliothecae nationalis [Romae], qui lectoribus in ipsa bibliotheca praesto est; equidem putaverim fuisse monasterii Sancti Salvatoris de Septimo, cum similissimus sit codicibus Sessorianis 6 et $7^{\prime 226}$. Scrittura minuscola carolingia. Abbreviazioni comuni. Pochissime correzioni.

73. Vallicellanus Tomus III, XII o XIII s., 450 x 305 mm., 246 fogli a due colonne, 34 righe di testo su ogni colonna e ca. 26 lettere in ogni riga. La Vita comincia al foglio $234 \mathrm{v}$ : Incipit uita beati pauli pmi $h[$ er]emite, e finisce al foglio $238 \mathrm{v}$ : [ ...] cum regnis suis. Il codice è conservato nella "Biblioteca Vallicelliana" a Roma. La provenienza è sconosciuta. Così lo descrive Poncelet: "Constat tribus codicibus mutilis simulque compactis, quorum prior (ff. 1-164) scriptura beneventana exaratus est saec. XI, alter (ff. 165-244) saec. XII/XIII, tertius (ff. 245-254) saec. XIII manu comuni sunt descripti, omnes quidem paginis bipartitis. Inter folia 43 et $44^{2}$ inseritus est quaternis minor (295 x 210 $\mathrm{mm}$.) foliorum 8 , saec. XI/XII lineis plenis manu beneventana rescriptus" ${ }^{27}$. La Vita viene divisa, da un correttore posteriore, nel seguente modo: prima manu: cap. I - Inter multos [ ...] nulli hominum compertum habetur (cioè dalla parola $\mathrm{n}^{\circ} 1$ alla parola $\mathrm{n}^{\circ} 179$ ); cap. II - Sub Decio et Valeriano persecutoribus [ ...] ac sic libidinis sensum succedens doloris magnitudo superavit (cioè dalla parola $\mathrm{n}^{\circ} 180$ alla parola $\mathrm{n}^{\circ} 392$ ); cap. III - Per idem ergo tempus quo talia gerebantur $[. .$.$] ea tempestate$ qua Cleopatrae junctus est Antonius (cioè dalla parola $\mathrm{n}^{\circ} 393$ alla parola $\mathrm{n}^{\circ}$ 599); cap. IV - Igitur adamato (quasi quod a Deo sibi offerretur) habitaculo [ ...] istam quoque gignat bestiam, incertum habemus (cioè dalla parola $\mathrm{n}^{\circ} 600$ alla parola $\mathrm{n}^{\circ} 881$ ); cap. V - Stupens itaque Antonius [...] Antiochiam ut ab Imperatore videretur, allatum est (cioè dalla pa-

25 A. Poncelet, Catalogus codicum hagiographicorum Latinorum Bibliothecarum Romanorum praeter quam Vaticanae, Bruxelles 1909, 117.

26 Poncelet, Catalogus codicum, 98.

27 Poncelet, Catalogus codicum, 300. 
rola $\mathrm{n}^{\circ} 882$ alla parola $\left.\mathrm{n}^{\circ} 1116\right)$; cap. VI - Sed ut propositum persequar [...] militibus suis Christus duplicavit annonam (cioè dalla parola $\mathrm{n}^{\circ}$ 1117 alla parola $\mathrm{n}^{\circ}$ 1467); cap. VII - Igitur Domino gratiarum actione celebrata $[. .$.$] Christo debitum spiritum redderet (cioè dalla parola \mathrm{n}^{\circ}$ 1468 alla parola $\left.\mathrm{n}^{\circ} 1865\right)$; cap. VIII - Cumque iam dies alia illuxisset [...] cum poenis suis (cioè dalla parola $n^{\circ} 1866$ alla parola $n^{\circ} 2398$, ossia sino alla fine del testo); secunda manu: cap. I - Inter multos [ ...] nulli hominum compertum habetur (cioè dalla parola $\mathrm{n}^{\circ} 1$ alla parola ${ }^{\circ}$ 179); cap. II - Sub Decio et Valeriano persecutoribus [ ...] ac sic libidinis sensum succedens doloris magnitudo superavit (cioè dalla parola $\mathrm{n}^{\circ} 180$ alla parola $\mathrm{n}^{\circ} 392$ ); cap. III - Per idem ergo tempus quo talia gerebantur [...] ea tempestate qua Cleopatrae junctus est Antonius (cioè dalla parola $\mathrm{n}^{\circ} 393$ alla parola $\mathrm{n}^{\circ}$ 599); cap. IV - Igitur adamato (quasi quod a Deo sibi offerretur) habitaculo [ ...] ad quem visendum deberet proficisci (cioè dalla parola $\mathrm{n}^{\circ} 600$ alla parola $\mathrm{n}^{\circ} 751$ ); cap. V - Illico erumpente luce [...] istam quoque gignat bestiam, incertum habemus (cioè dalla parola $\mathrm{n}^{\circ} 752$ alla parola $\mathrm{n}^{\circ} 881$ ); cap. VI - Stupens itaque Antonius [...] et in universam terram exiit sonus eius (cioè dalla parola $\mathrm{n}^{\circ} 882$ alla parola $\mathrm{n}^{\circ} 998$ ); cap. VII - Talia eo loquente, longaevus viator ubertim faciem lacrimis rigabat [ ...] Antiochiam ut ab Imperatore videretur, allatum est (cioè dalla parola $\mathrm{n}^{\circ} 999$ alla parola ${ }^{\circ} 1116$ ); cap. VIII - Sed ut propositum persequar [ ...] militibus suis Christus duplicavit annonam (cioè dalla parola $\mathrm{n}^{\circ} 1117$ alla parola $\mathrm{n}^{\circ} 1467$ ); cap. IX - Igitur Domino gratiarum actione celebrata [ ...] immo terrae terram reddas (cioè dalla parola $\mathrm{n}^{\circ} 1468$ alla parola $\mathrm{n}^{\circ} 1596$ ); cap. $\mathrm{X}-H$ is Antonius auditis, flens et gemens [...] Christo debitum spiritum redderet (cioè dalla parola $\mathrm{n}^{\circ} 1597$ alla parola $\mathrm{n}^{\circ} 1865$ ); cap. XI - Cumque jam dies alia illuxisset [ ...] extremum halitum fundam (cioè dalla parola $\mathrm{n}^{\circ} 1866$ alla parola $\left.\mathrm{n}^{\circ} 2045\right)$; cap. XII - Talia eo animo volvente [...] cum poenis suis (cioè dalla parola $\mathrm{n}^{\circ} 1866$ alla parola $\mathrm{n}^{\circ} 2398$, ossia sino alla fine del testo). Scrittura comune molto bella. Abbreviazioni comuni. Pochissime correzioni.

92. Vaticanus Lat. $600^{28}$, XIV s., $490 \times 343 \mathrm{~mm} ., 170$ fogli a due colonne, 43 righe di testo su ogni colonna, e in ogni riga ca. 30 lettere. La Vita comincia al foglio 165: Vita uel obitus sĉi pauli pmi hêmite, edita a sĉo Ieronimo $p b^{\circ} \circ$, e finisce al foglio $167 \mathrm{v}$ : [ ...] cum regnis suis. Il codice è conservato nella "Biblioteca Apostolica Vaticana". Nel 1422, apparteneva al monastero di San Gregorio a Roma ${ }^{29}$, dove probabilmente fu copiato.

28 A proposito di questo codice, cf. Limone, La tradizione, p. 895; Morales, Edición crítica, p. 125 e passim.

29 Cf. fol. $158 \mathrm{v}$. 
Esso "constat simul compactis duobus voluminibus (F. 1-115 et F. 116171), quae a singulis librariis sunt descripta" ${ }^{30}$. La scrittura è una gotica italiana. Le abbreviazioni sono frequenti. Parecchie correzioni e abrasioni.

103. Vaticanus Lat. $5257^{31}$, XV s., 201 x $131 \mathrm{~mm}$., 61 fogli a due colonne e 54 righe di testo su ogni colonna e ca. 35 lettere in ogni riga. La Vita comincia al foglio 43: Inter multos [ ...], e finisce al foglio 49: [ ... cum regnis suis. Il codice è custodito nella "Biblioteca Apostolica Vaticana". Così lo descrive Poncelet: "Constat simul compactis aliquot codicibus exaratis saec. XV et XVI. I. folia 1-8 memb. $\left(0^{\mathrm{m}}, 203 \times 0,138\right)$ exar. s. XIV/XV. II. f. 8-29, olim sign. 1-21, ex charta, $\left(0^{\mathrm{m}}, 207\right.$ x 0,143$)$, exar. a. 1506. III. f. 30-38, ex charta, $\left(0^{\mathrm{m}}, 209 \times 0,138\right)$ XV s. IV. f. 39-49, memb. $\left(0^{\mathrm{m}}, 201 \times 0\right.$, 131) XV s. V. f. A, B et sign. 4-13, ex charta, $\left(0^{\mathrm{m}}, 208 \times\right.$ x 0, 155)"32. A proposito della provenienza, leggiamo al foglio B v: Scripto e minato per mano di me Feliciano da Verona a mio nome e instantia negli anni di Christo 1460 dil mese di zenaro. Non è certo, però, se il codice sia scritto colà. La scrittura è una gotica brutta e poco leggibile, con tantissime abbreviazioni. Le correzioni sono abbastanza frequenti.

109. Vaticanus Lat. $9309^{33}$, XIII s. (inizio), 307 x 225 mm., 75 fogli (sui fogli 44-75 troviamo due colonne) con 39 righe di testo e ca. 34 lettere in ogni riga. La Vita non è completa ed inizia, al foglio 68, con la parola $\mathrm{n}^{\circ} 1760$ : suū ĉfecto itinero $p$ [er]uenit, e finisce al foglio $68 \mathrm{v}$ : [ ...] cum regnis suis. Il codice è custodito nella "Biblioteca Apostolica Vaticana". La provenienza è sconosciuta. La scrittura è una gotica francese regolare. Le abbreviazioni sono frequenti. Una sola correzione, probabilmente dallo stesso scrivano.

110. Vaticanus Lat. $13393^{34}$, IX o X s., 352 x $240 \mathrm{~mm}$. Contiene otto quaderni. La Vita si trova nel quaderno sesto, intitolato (con una mano del

30 A. Poncelet, Catalogus codicum hagiographicorum Latinorum Bibliothecae Vaticanae, Bruxelles 1910, 22.

31 A proposito di questo codice, cf. R. Massigli, Un manuscrit inédit de l'Évangile du Pseudo-Mathieu, "Mélanges de l'École Française de Rome" 33 (1913) 83; J. Gijsel, Zu welcher Textfamilie des Pseudo-Matthäus gehört die Quelle von Hrotsvits Maria?, CM 32 (1971-1980) 280; Morales, Edición crítica, p. 127 e passim.

32 Poncelet, Catalogus [ ...] Bibliothecae Vaticanae, p. 129.

33 A proposito del codice, cf. Degórski, $\mathrm{La}$ "conlatio [ ...] sino alle parole: “[...] incitata sunt [ ...], pp. 57-101; Degórski, La "conlatio [ ...] sino alle parole: “[ ...] pro Christi nomine [ ...], pp. 839-866; Degórski, La "conlatio [ ...] dalle parole: "Amathas [ ...], pp. 119-164; Degórski, La "conlatio [ ...] gladio percuti” dello stesso capitolo, pp. 839866; Degórski, La "conlatio [ ...] dalle parole: "Verum hostis [ ...], pp. 251-271; Morales, Edición crítica, p. 128 e passim; Degórski, I manoscritti delle biblioteche di Roma, p. 292.

34 A proposito del codice, cf. M. Oberleitner, Die handschriftliche Überlieferung der Werke des Heiligen Augustinus, Wien 1969 = "Sitzungsberichte des Österreichischen Akademie der Wissenschaften. Philosophisch-Historische Klasse" 263 (1969) 399; 
XX secolo): Le fogli di un passionario o delle vitae SS. patrum. Sec. X. ed un frammento di altri due fogli, ed un piccolo frammento staccato. Le pagine prime ed ultime di ogni paio di fogli sono illeggibili. La Vita non è completa ed inizia al foglio $26 \mathrm{v}$ con la parola $\mathrm{n}^{\circ} 275$ : [mu]scarum [ ...], e finisce al foglio 27 con la parola $\mathrm{n}^{\circ} 761$ : [ ...] sustent $[$ ans]. Il codice è custodito nella "Biblioteca Apostolica Vaticana". La provenienza è sconosciuta. La scrittura è una minuscola carolingia. Abbreviazioni ordinarie. Poche correzioni.

111. Vaticanus Barberinianus Lat. $469^{35}$, XV s., 210 x 154 mm., 159 fogli con 35 righe di testo e ca. 47 lettere in ogni riga. La Vita comincia al foglio 61: Hieronymus in Vitam Beati pauli primi heremitae. Incipit feliciter, e finisce al foglio 65: Unde grās agam'oēs oīpotenti dō qui tanta mirabilia corruscare dignatus est $\bar{l}$ sctīs suis: ut ip [s] o adiunante meream[ur] eor $[u m]$ adiuuare ${ }^{36} \dot{p}$ cibus. Amen. Il codice è custodito nella "Biblioteca Apostolica Vaticana". La provenienza è sconosciuta. Scrittura gotica francese. Abbreviazioni frequenti. Molte correzioni.

132. Vaticanus Urbinas Lat. 396 (già: 743) ${ }^{37}$, XV s., 333 x 228 mm., 207 fogli con 39 righe di testo e ca. 56 lettere in ogni riga. La Vita comin-

Degórski, La "conlatio [ ...] sino alle parole: “[ ...] incitata sunt [ ...], pp. 57-101; Degórski, La "conlatio [ ...] sino alle parole: “[ ...] pro Christi nomine [ ...], pp. 839866; Degórski, La "conlatio [ ...] dalle parole: "Amathas [ ...], pp. 119-164; Degórski, La "conlatio [ ...] gladio percuti" dello stesso capitolo, pp. 839-866; Degórski, La "conlatio [ ...] dalle parole: "Verum hostis [ ...], pp. 251-271; Degórski, I manoscritti delle biblioteche di Roma, p. 292.

35 A proposito del codice, cf. C. Lambot, L'homélie du Pseudo-Jérôme sur l'Assomption et l'évangile de la Nativité de Marie d'après une lettre inédite d'Hincmar, RBen 46 (1934) 276; Oberleitner, Die handschriftliche Überlieferung, pp. 245, 254-255, 306, 319; Oberleitner, Die handschriftliche Überlieferung der Werke des Heiligen Augustinus, Wien 1970 = "Sitzungsberichte des Österreichischen Akademie der Wissenschaften. Philosophisch-Historische Klasse" 267 (1970) 298; J. Divjak - F. Römer, Ergänzungen zur Bibliotheca Hieronymiana manuscripta. I: Dänemark, Großbritannien, Irland, Italien, Polen, Portugal, Schweden, Spanien, Vatikan, "Scriptorium" 30 (1976) 95, 98, 101; Morales, Edición crítica, passim.

36 Prima manu: adiuuare. Secunda manus in "adiunari" correxit.

37 A proposito di questo codice, cf. Bethmann, Nachrichten, p. 263; Wilmart, Les rédactions, pp. 225-234, 240, 244; Dolbeau -Lambert - Lévy - Petitmengin - Tilliette, Les Vies latines de sainte Pélagie, p. 300; Dolbeau - Lévy - Petitmengin - Rothschild Tilliette, Les Vies latines de Sainte Pélagie, p. 291; M.P. Ciccarese, Le visioni di S. Fursa, "Romano Barbarica" 8 (1984-1985) 248; Degórski, La "conlatio codicum" della "Vita S. Pauli Primi Eremitae” (dall'inizio del primo capitolo sino alle parole: "[ ...] incitata sunt studia", pp. 57-101; Degórski, Conlatio codicum della "Vita Sancti Pauli Primi Eremitae" di San Girolamo (dalle parole: "Amathas, pp. 119-162; Degórski, La "conlatio codicum" della "Vita S. Pauli Primi Eremitae" di Girolamo, dall'inizio del II capitolo, pp. 839-866; Degórski, La "conlatio codicum” della "Vita S. Pauli Primi Eremitae” di 
cia al foglio 2: INTER MVLTOS [ ...], e finisce al foglio $4 \mathrm{v}:$ EXPLICIT VITA BEATI PAVLI MONACI QVI FVIT ANTE SANCTUM ANTONIV[M]. QVEM IP[S]E SEPELLIVIT IN HEREMO HIC NV[M]Q[VAM] FVIT IN CIVITATIBVS POSTQ[AM] IN SILITVDINE DEMORATUS EST. Il codice è custodito nella "Biblioteca Apostolica Vaticana". La provenienza è sconosciuta. La scrittura è un'umanistica piuttosto primitiva. Le abbreviazioni sono frequenti e difficili. Nessuna correzione.

135. Basilicanus A. $4^{38}$, XI s., $472 \times 340 \mathrm{~mm}$., 238 fogli a due colonne. La Vita comincia al foglio 223: INCIP[IT] UITA SCI PAULI MONACHI. Kl $i u l$., e finisce al foglio 227 con un'unica parola aggiunta al testo geronimiano: init $^{39}$. Si trova nell" "Archivio del Capitolo di San Pietro in Vaticano". La provenienza è sconosciuta. Scrittura carolingia. Abbreviazioni comuni. Tantissime correzioni e abbrasioni.

\section{Un tentativo di classificazione dei codici}

Lo Iagellonicus Cracoviensis 2265 AA XII 9 è molto vicino alle famiglie "I" e "Q". Infatti, con la famiglia "I" condivide le seguenti lezioni, caratteristiche a questo gruppo:

\begin{tabular}{|c|c|c|c|}
\hline Parola $n^{\circ}$ & PL & Varianti & Mss. concordanti \\
\hline 1. $403-404$ & cum sorore & cum sorore $<$ sua $>^{40}$ & $\begin{array}{c}23 \text { a; "I": } 29,30,31,32,33,41,60,61, \\
64,68,71,71 \text { a, } 74,78,86,87,88,90, \\
95,101,102,115,117,123,133,137, \\
140\end{array}$ \\
\hline 2. 1281 & recipis & recipis $^{41}$ & $\begin{array}{l}23 \text { a; "I": } 29,30,31,32,33,41,60,61, \\
64,68,71,71 \text { a, 78, 86, 87, 88, 90, 95, } \\
101,102,115,117,123,133,137,140\end{array}$ \\
\hline
\end{tabular}

Girolamo, dalle parole: "Verum hostis, pp. 251-271; Morales, Edición crítica, passim; Degórski, I manoscritti delle biblioteche di Roma, pp. 297-298.

38 A proposito del codice, cf. Paschini, Ricerche, p. 177; De Marco, Censimento, p. 1104; J. Gardner, The Stefaneschi Altarpiece: A Reconsideration, "The Journal of the Warburg and Courtauld Institutes" 37 (1974) 58, 66; Weismann, Die "Passio, pp. 31-43; Dolbeau, Le légendier de la cathédrale, p. 125; Supino Martini - Petrucci, Materiali ed ipotesi, p. 55; Verrando, Liberio-Felice, p. 125; J.E. Cross, The "Passio s. Laurentii et aliorum": Latin Manuscripts and the "Old English Martyrology", "Mediaeval Studies" 45 (1983) 200; Tomea, L'agiografia milanese, pp. 640-641.

39 "Finit" secunda manus, ut videtur, delevit.

40 La stessa aggiunta è anche presente nei manoscritti che non appartengono alla famiglia "I": Vaticanus Burghesianus Lat. 160 (si tratta qui del primo testo della Vita presente in questo codice), Vaticanus Chisianus Lat. H IV 119, Vallicellanus Codex D. 2. 
Con la famiglia "Q", invece, condivide la seguente lezione, caratteristica a questo gruppo:

\begin{tabular}{|c|c|c|c|}
\hline Parola n $^{\circ}$ & PL & Varianti & Mss. concordanti \\
\hline 3.817 & inquit & inquit $<$ Antonius $>42$ & $\begin{array}{c}23 \text { a: "Q": } 62,65,77,75,94,97,98,99,105, \\
107,106,113,114,120,128,129,130,131\end{array}$ \\
\hline
\end{tabular}

Basandoci sul numero delle lezioni caratteristiche delle famiglie "I" e "Q", possiamo affermare che il nostro codice si avvicina piuttosto al gruppo "I", perché condivide con esse due caratteristiche lezioni. Parlando più precisamente, il codice presenta una più grande vicinanza a quel sottogruppo della famiglia "I" che è composto dai codici: Angelicus 234, Casanatensis Codex 253 (A. IV. 40), Iesuiticus 973 (3102), Vallicellanus Codex C. 74, Vaticanus Lat. 344 (2331), Vaticanus Lat. 349 (2341), Vaticanus Lat. 358 (2367), Vaticanus Lat. 363 (3017), Vaticanus Lat. 5087, Vaticanus Lat. 5119 (5181), Vaticanus Chisianus Lat. A IV 90, Vaticanus Palatinus Lat. 860. Ecco le lezioni che lo Iagellonicus condivide con questi codici della famiglia "I":

\begin{tabular}{|c|c|c|c|}
\hline 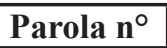 & PL & Varianti & Mss. concordanti \\
\hline 4. $23-24$ & $\begin{array}{l}\text { sumpsere } \\
\text { principium }\end{array}$ & sumpsere principia ${ }^{43}$ & $\begin{array}{c}23 \text { a; "I": } 61,68,78,86,88,90, \\
102,117\end{array}$ \\
\hline 5.420 & sexdecim & quindecim ${ }^{44}$ & $\begin{array}{c}23 \text { a; "I": } 61,68,78,86,87,88,90, \\
101,102,117\end{array}$ \\
\hline 6. $874-875$ & $\begin{array}{l}\text { animalium } \\
\text { ferax }\end{array}$ & formas animalium $^{45}$ & $\begin{array}{c}23 \text { a; "I": } 60,61,68,86,88,90 \\
102,117\end{array}$ \\
\hline
\end{tabular}

${ }^{41}$ La stessa lezione è anche presente nei manoscritti che non appartengono alla famiglia "I": Bruxellensis 64; Laurentianus pl. 19, cod. 16; Casanatensis Codex 588 (B. V. 34), Casanatensis Codex 718 (B. I. 3), Casanatensis Codex 1898 (B. II. 15), Vallicellanus Codex C. 55, Vallicellanus Tomus III, Vallicellanus Tomus VI, Vaticanus Lat. 1200 (2188), Vaticanus Lat. 5257, Vaticanus Lat. 6933, Vaticanus Barberinianus Lat. 605, Vaticanus Barberinianus Lat. 621, Vaticanus Burghesianus Lat. 160 (si tratta qui del primo testo della Vita Sancti Pauli Primi Eremitae che si trova in questo codice), Vaticanus Urbinas Lat. 51 (107), Vaticanus Urbinas Lat. 389 (742), Vaticanus Lat. 1201 (71), Vaticanus Palatinus Lat. 179, Vaticanus Rossianus Lat. 287.

42 Il codice Iagellonicus Cracoviensis 2265 AA XII 9 legge: inquit < anthonius>.

43 Il codice Iagellonicus Cracoviensis 2265 AA XII 9 legge: sumsere principia. Tale lezione è anche presente nei manoscritti che non appartengono alla famiglia "I": Vaticanus Lat. 5257; Vaticanus Chisianus Lat. H IV 119.

44 La stessa lezione è anche presente nel Vaticanus Chisianus Lat. H IV 119 che non fa parte della famiglia "I".

45 Il codice Angelicus 234 (indicato qui con il numero 60) legge: forma animalium. 


\begin{tabular}{|c|c|c|c|}
\hline Parola $^{\circ}$ & PL & Varianti & Mss. concordanti \\
\hline 7. $910-911$ & pars corporis & corporis pars & 23 a; "I": 101, 123 \\
\hline 8.1333 & adveneris & adueneris $<$ sepelire $>46$ & $\begin{array}{c}23 \text { a; "I": } 60,61,64,68,78,86,87, \\
88,90,101,102,117,123\end{array}$ \\
\hline 9. 1810 & ex & de & $\begin{array}{c}23 \text { a; "I": } 60,61,68,78,86,87,88, \\
90,101,102,117\end{array}$ \\
\hline 10. 1827 & loquenti & $\begin{array}{c}\text { tacendi }<\text { tempus } \\
\text { plangendi et tempus } \\
\text { ridendi }>47\end{array}$ & $\begin{array}{c}23 \mathrm{a} ; \text {;"I": } 60,61,78,86,88,90,101, \\
102,117,123\end{array}$ \\
\hline 11. 2304 & illi & illi $<$ quidem $>^{48}$ & 23 a; "I": 60, 87, 101, 117, 123 \\
\hline
\end{tabular}

Queste varianti dimostrano una più stretta vicinanza che intercorre tra lo Iagellonicus e quei codici che formano il sottogruppo della famiglia "I". Potremmo, quindi, cercare di annoverarlo proprio a tale sottogruppo. Pare, però, che le somiglianze siano risultato di tante contaminazioni alle quali fu soggetto il testo della Vita. Infatti, il codice possiede anche lezioni che troviamo nei codici del seguente sottogruppo della famiglia "Q": Casanatensis Codex 588 (B. V. 34), Casanatensis Codex 1898 (B. II. 15), Vallicellanus Tomus VI, Vallicellanus Codex C. 55, Vaticanus Lat. 1199 (2598), Vaticanus Lat. 1200 (2188), Vaticanus Lat. 1201 (71), Vaticanus Lat. 6933, Vaticanus Barberinianus Lat. 605, Vaticanus Barberinianus Lat. 621, Vaticanus Palatinus Lat. 179, Vaticanus Rossianus Lat. 287, Vaticanus Urbinas Lat. 48 (102), Vaticanus Urbinas Lat. 51 (107). Ecco, ad esempio, alcune lezioni communi:

46 La stessa aggiunta è presente nei codici che non appartengono al gruppo "I": Casanatensis Codex 1898 (B. II. 15), Vallicellanus Codex C. 55, Vallicellanus Tomus III, Vaticanus Lat. 1199 (2598), Vaticanus Lat. 1200 (2188), Vaticanus Barberinianus Lat. 469, Vaticanus Chisianus Lat. H IV 119. I codici: Angelicus 234, Iesuiticus 973 (3102), Vallicellanus Codex C. 74, Vaticanus Lat. 358 (2367), Vaticanus Lat. 363 (3017) leggono: adueneris $<$ sepellire $>$.

47 La stessa aggiunta è presente nei codici che non appartengono al gruppo "I": Vaticanus Lat. 5257, Vaticanus Barberinianus Lat. 469. Quest'ultimo codice e il codice Vaticanus Palatinus Lat. 860 hanno la seguente aggiunta: tempus ridendi e tempus plangendi. Il codice Vallicellanus Codex C. 74 ha l'aggiunta: et tempus plangendi et tempus ridendi. Il menzionato codice Vaticanus Lat. 5257, che non appartiene al gruppo "I", ha l'aggiunta: tempus ridendi. Tempus plangendi. Infine, i codici: Angelicus 234, Vaticanus Lat. 5087 e Vaticanus Lat. 5119 (5181), che appartengono al gruppo "I", hanno la seguente aggiunta: tempus plangendi. Tempus ridendi.

48 La stessa aggiunta è presente nel Vaticanus Lat. 5257, che non appartiene al gruppo "I". 


\begin{tabular}{|c|c|c|c|}
\hline Parola $^{\circ}$ & PL & Varianti & Mss. concordanti \\
\hline 12. 109 & tulit & tulit $<$ legendi $>49$ & $\begin{array}{l}23 \text { a; “Q”: } 62,65,77,97,98,99 \\
107,114,120,128,129,130,131\end{array}$ \\
\hline 13.179 & habetur & uidetur $^{50}$ & 23 a; “Q”: 99, $114,120,128,129$, \\
\hline 14. 854 & volucri & uolucri $<$ se $>51$ & 23 a; “Q": 99, $113,120,128,129$, \\
\hline 15.973 & colit & $\begin{array}{c}\text { colit. }<\text { Et antonius: } \\
\text { Vbi uadis? Respon- } \\
\text { dit }>^{52} \\
\end{array}$ & $\begin{array}{c}23 \text { a; " Q": } 62,65,77,97,98,99 \\
113,120,128,129,131\end{array}$ \\
\hline 16.978 & precamur & precamur $<$ ergo $>^{53}$ & $\begin{array}{l}23 \text { a; “Q”: } 62,65,77,97,98,99 \\
107,113,120,128,129,130,131\end{array}$ \\
\hline 17. 1059 & loquuntur & colunt $^{54}$ & $\begin{array}{c}23 \text { a; “Q”: 65, 99, 113, 120, 128, } \\
129,131\end{array}$ \\
\hline 18. 1478 & consedit & consederunt $t^{55}$ & $\begin{array}{c}23 \text { a; “Q”: } 62,65,75,77,97,98,99 \\
105,113,114,120,128,129,130\end{array}$ \\
\hline
\end{tabular}

49 La stessa aggiunta è presente nei codici che non fanno parte della famiglia "Q": Monacensis 22033, Vaticanus Lat. 1188 (234).

50 La stessa lezione è presente nei codici che non fanno parte della famiglia "Q": Farfensis 5 (270), Basilicanus A. 2, Basilicanus A. 4.

51 La stessa aggiunta è presente nei manoscritti che non appartengono alla famiglia “Q": Casanatensis Codex 726, Vallicellanus Tomus III, Vaticanus Palatinus Lat. 860. Quest'ultimo codice e i Vaticanus Lat. 1201 (71), Vaticanus Rossianus Lat. 287, Vaticanus Urbinas Lat. 48 (102) leggono: uolucri <sese>. Il Vaticanus Urbinas Lat. 389 (742), invece, legge: uolucri $<$ sit $>$.

52 Le stesse aggiunte sono presenti nei manoscritti che non appartengono alla famiglia “Q": Bruxellensis 64, Angelicus 234, Vallicellanus Tomus III, Vaticanus Lat. 5411, Vaticanus Urbinas Lat. 1503 (108). Il codice Angelicus 234 legge: <Et antonius quo uadis ait; respondet>. Il codice Vallicellanus Tomus III, invece, presenta l'aggiunta: $<$ Cui antonius. Quo uadis? Respondit>. Il Vaticanus Urbinas Lat. 1503 (108), infine, possiede: $<$ Quo uadis iquit?>. I manoscritti, invece, Casinensis 146, Casinensis 463, Vaticanus Burghesianus Lat. 160 (si tratta qui del primo testo della Vita contenuto in questo codice), che non appartengono alla famiglia "Q", hanno la seguente aggiunta: <quo uadis $>$. I codici, invece, Casanatensis Codex 588 (B V 34), Casanatensis Codex 1898 (B II 15), Vaticanus Lat. 1199, Vaticanus Lat. 1200 (2188), Vaticanus Barberinianus Lat. 605, Vaticanus Urbinas Lat. 389 (742), che appartengono alla famiglia “Q", leggono: <Et antonius. Quo uadis? Respondit>.

53 L'aggiunta è presente nei codici che non appartengono alla famiglia "Q": Monacensis 22033, Vaticanus Lat. 1188 (234). Il Casanatensis Codex 588 (B V 34) che appartiene al gruppo "Q", legge: precamur $<$ ergo te $>$.

54 La stessa variante è presente nel Vaticanus Lat. 5257 che non appartiene al gruppo "Q".

55 La stessa variante è presente nei manoscritti che non appartengono alla famiglia “Q”: Parisinus Lat. 5342, Parisinus Lat. 16050, Vaticanus Barberinianus Lat. 469. 


\begin{tabular}{|c|c|c|c|}
\hline Parola $n^{\circ}$ & PL & Varianti & Mss. concordanti \\
\hline 19. 1999 & decantans & cantatis $<$ expleuit $>56$ & $\begin{array}{c}23 \text { a; "Q": } 62,65,99,113,120,128, \\
129,131\end{array}$ \\
\hline 20. 2047-2048 & eo animo & eo $<$ in $>$ animo $^{57}$ & $\begin{array}{c}23 \text { a; "Q": } 62,65,75,77,97,98,99, \\
107,113,114,120,128,129,131\end{array}$ \\
\hline
\end{tabular}

Queste varianti possono indicare una vicinanza che intercorre tra lo Iagellonicus e il suddetto sottogruppo della famiglia "Q". Non possiamo, però, annoverare lo Iagellonicus alla famiglia "Q", perché esso, essendo tardivo, è frutto di diverse contaminazioni. Eccone alcune prove:

\begin{tabular}{|c|c|c|c|}
\hline Parola $n^{\circ}$ & PL & Varianti & Mss. concordanti \\
\hline 21.252 & perseverantem & $\begin{array}{c}<\text { igitur }>\text { perseueran- } \\
\text { tem }>^{58}\end{array}$ & 23 a; “D”: $35,40,85$ \\
\hline 22.272 & reponi & resupinari & $\begin{array}{c}23 \text { a; "D": 52, 119; “E”: } 12,36 ; \\
\text { "L": } 116 ; \text { "Z": 16, 22, } 49\end{array}$ \\
\hline 23.702 & annos & anno $<$ etatis sue $>59$ & $23 \mathrm{a} ;$ "D": 85 \\
\hline 24.964 & vario & uano & 23 a; "D": 35,40 \\
\hline 25.1853 & complectens & contemplans & $23 \mathrm{a} ; 26,92$ \\
\hline 26. 2153-2154 & muta quoque & muta & $\begin{array}{c}23 \text { a; "D": } 52,119 ; \text { "E": 39; } \\
\text { "J": } 1,21 ; \text { "K": } 24\end{array}$ \\
\hline 27.2292 & texitis & contextis & $23 \mathrm{a} ; " \mathrm{E} ": 20,49$ \\
\hline
\end{tabular}

Possiamo constatare che lo Iagellonicus è molto simile ai codici delle famiglie "I" e "Q". Potrebbe essere quasi annoverato ad una di esse, ma purtroppo, essendo molto tardivo, è frutto di diverse contaminazioni.

Vediamo ora altri codici inclassificabili. I Casanatensis Codex 718 (B. I. 3), Vaticanus Lat. 7014 e Vaticanus Barberinianus Lat. 469 hanno in comune molte lezioni peculiari, fra cui:

56 Il Vallicellanus Lat. Tomus III, che non appartiene al gruppo "Q", legge: cantatis <funus expleuit>. La stessa lezione è presente nei codici che appartengono alla famiglia "Q": Casanatensis Codex 588 (B. V. 34), Vaticanus Lat. 1201 (71), Vaticanus Barberinianus Lat. 605, Vaticanus Rossianus Lat. 287, Vaticanus Urbinas Lat. 48 (102), Vaticanus Urbinas Lat. 389 (742). Lo Iagellonicus Cracoviensis 2265 AA XII 9, invece, legge: cantatis <expletis>.

57 La stessa aggiunta è presente nei manoscritti che non appartengono alla famiglia “Q”: Iesuiticus 973 (3102), Casanatensis Codex 253 (A. IV. 40), Vallicellanus Tomus III, Vallicellanus Codex C. 74, Vaticanus Lat. 358 (2367), Vaticanus Lat. 363 (3017), Vaticanus Lat. 600 (153), Vaticanus Lat. 1188 (234), Vaticanus Lat. 5257, Basilicanus A. 4.

58 Il codice Iagellonicus Cracoviensis 2265 AA XII 9 legge: $<$ unum > perseuerantem.

59 Lo Scaphusianus 104, che appartiene al gruppo "D", legge: anno <ętatis suę>. A proposito di questo codice, cf. Morales, Edición crítica, passim. 


\begin{tabular}{|c|c|c|c|}
\hline 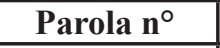 & PL & Varianti & Mss. concordanti \\
\hline 28.8 & potissimum & potissimo & 63,108 \\
\hline 29. $408-411$ & $\begin{array}{l}\text { post mortem am- } \\
\text { borum parentum }\end{array}$ & --- & 63,108 \\
\hline 30.86 & --- & $<$ qui $>60$ & $63,108,111$ \\
\hline 31. 171-172 & et quas & atque $^{61}$ & $63,108,111$ \\
\hline 32.251 & subjicimus & subiciamus $^{62}$ & 63,108 \\
\hline 33.355 & --- & $<\operatorname{sic}>$ & $63,108,111$ \\
\hline 34. $377-379$ & $\begin{array}{l}\text { praecisam mordi- } \\
\text { cus linguam }\end{array}$ & $\begin{array}{l}\text { precisa morsu lingua } \\
\text { precisam morsu linguam }\end{array}$ & $\begin{array}{c}63,108 \\
111\end{array}$ \\
\hline 35.426 & apprime & adplene $^{63}$ & 63,108 \\
\hline 36. $440-445$ & $\begin{array}{l}\text { remotiorem et se- } \\
\text { cretioremsecessit. } \\
\text { Verum quid }\end{array}$ & $\begin{array}{l}\text { remotiori et secretiori se con- } \\
\text { tulit. Verum }<\mathrm{ad}>\text { quid }^{64} \\
\text { remotiori et secretiori se con- } \\
\text { tulit. Verum quid }\end{array}$ & $\begin{array}{c}63,108 \\
111\end{array}$ \\
\hline $37.2344-2346$ & vos, parcite saltem & --- & 63,108 \\
\hline 38.2398 & suis. & $\begin{array}{l}\text { suis. }<\text { Vnde gratias agamus } \\
\text { omnes omnipotenti deo, qui } \\
\text { tanta mirabilia coruscare dig- } \\
\text { natus est in sanctis suis, ut ipso } \\
\text { adiuuante mereamur eorum } \\
\text { saluari precibus. Amen }>65\end{array}$ & $63,108,111$ \\
\hline
\end{tabular}

Queste varianti mostrano una stretta parentela tra i codici: Casanatensis Codex 718 (B. I. 3), Vaticanus Lat. 7014 e Vaticanus Barberinianus Lat. 469. Tutti e tre hanno lezioni caratteristiche del gruppo "R", ma anche alcune varianti prioprie del gruppo " $\mathrm{D}$ ". Potrebbero quasi essere classificati in "D", ma pare che le somiglianze siano causate da molte contaminazioni. Un esame più preciso dei Casanatensis Codex 718 (B. I. 3) e Vaticanus Lat. 7014 rivela le seguenti differenze:

60 La stessa aggiunta è presente nei: Lateranensis Codex A. 79, Vaticanus Barberinianus Lat. 621, Vaticanus Urbinas Lat. 51 (107), Basilicanus B. 50.

61 La stessa aggiunta è presente nei: Vaticanus Burghesianus Lat. 160 (si tratta qui del primo testo della Vita contenuto in questo codice), Rotomagensis U 108.

${ }^{62}$ La stessa aggiunta è presente nei: Vaticanus Urbinas Lat. 1503 (108), Basilicanus B. 50 .

63 La stessa aggiunta è presente nei: Veronensis XXXVIII (36), Cotton Caligula A XV.

64 La stessa lezione si trova nel Lateranensis Codex A. 79. L'aggiunta, però, $\langle a d\rangle$ fu scritta a secunda manu, ut videtur, super lineam. Una simile lezione (remotiori et secretiori $<$ et remotiori $>$ se contulit. Verum $<a d>$ quid) è presente nel Vallicellanus Tomus III.

65 Al posto della parola saluari, il Vaticanus Barberinianus Lat. 469 presenta: adiuuari. 


\begin{tabular}{|c|c|c|c|}
\hline Parola $^{\circ}$ & PL & Il Casanatensis Codex 718 & Il Vaticanus Lat. 7014 \\
\hline 39.38 & coepisse & cępisset & cępisse \\
\hline 40.42 & Alii & Alia & Alii \\
\hline $41.45-46$ & quam opinionem & qua opinionem & qua opinione \\
\hline 42.488 & adolescens & adholescens & adolescens \\
\hline 43.2302 & e & --- $^{66}$ & e \\
\hline
\end{tabular}

Tali differenze possono essere spiegate come sviste dello scrivano. Entrambi i codici concordano nell'ortografia e nella punteggiatura. Quindi, possiamo concludere che il Vaticanus Lat. 7014 è una semplice copia del Casanatensis Codex 718 (B. I. 3).

Il Lateranensis Codex A. 79 si avvicina primariamente alla famiglia "W" (in modo particolare al Basilicanus B. 50) e al gruppo "I" (soprattutto ai Casanatensis Codex 726 e Vallicellanus Codex C. 74), e secondariamente al gruppo "Q" (specialmente al Vaticanus Lat. 1199). Mostra anche una certa vicinanza con le famiglie "D", "L" e "K". Inoltre, è vicinissimo al Casanatensis Codex 718 (B. I. 3) (e, quindi, anche ai Vaticanus Lat. 7014 e Vaticanus Barberinianus Lat. 469) e, in secondo luogo, al Vallicellanus Tomus III.

Il Farfensis 5 (270) è vicino primariamente al gruppo "L" (in modo speciale al Cantabrigiensis Mm. 4. 28) ${ }^{67}$ ), e secondariamente alle famiglie "E" e "Z" (specialmente al Bruxellensis 9398/99, che appartiene alla famiglia "E", e al Parisinus Lat. 10840, che fa parte del gruppo "Z"). Per quanto riguarda le sue relazioni con i codici inclassificabili, è possibile rilevare una parentela con i Bruxellensis 4815/18 e Parisinus Lat. 9741.

I Sessorianus 5 (XXIX) e Vaticanus Barberinianus Lat. 586, che mostrano poca affinità con qualsiasi gruppo singolo, sono probabilmente più vicini alla famiglia " $Z$ ". Inoltre, essi sono molto vicini tra di loro e presentano alcune lezioni particolari esclusive, quali:

\begin{tabular}{|c|c|c|c|}
\hline Parola n $^{\circ}$ & PL & Varianti & Mss. concordanti \\
\hline $44.331-332$ & recedentibus cunctis & --- & 69,112 \\
\hline 45.504 & progrediens & prodiens & 69,112 \\
\hline 46.704-708 & vitam coelestem ageret in terris & $\begin{array}{c}\text { cęlestem in terris } \\
\text { ageret uitam }\end{array}$ & 69,112 \\
\hline
\end{tabular}

I codici Vallicellanus Tomus III e Vaticanus Lat. 5257 potrebbero essere classificati nella famiglia "I" (specialmente il Vaticanus Lat. 5257), ma le somiglianze sono probabilmente dovute a molti incroci (soprattutto per quanto riguarda il Vaticanus Lat. 5257).

\footnotetext{
66 La $e$ viene anche omessa nel Vaticanus Palatinus Lat. 844.

67 A proposito del Cantabrigiensis Mm. 4. 28, cf. Morales, Edición crítica, passim.
} 
Il Vaticanus Lat. 600 (153) si avvicina soprattutto al Vallicellanus Tomus VI del gruppo "Q", e ai Vaticanus Ottobonianus Lat. 12068, Parisinus Lat. 11749, Bruxellensis 207/8 e Cantabrigiensis 389, che appartengono alla famiglia "D". Presenta anche una certa affinità con i Sangallensis $558^{69}$ e Monacensis 17137, che appartengono al gruppo "E". Per quanto concerne, invece, l'affinità del manoscritto in questione con i codici inclassificabili, si ravvisa una vicinanza con il Vindobonensis Lat. 420.

Il Vaticanus Lat. 9309, essendo mutilo, è difficile da classificare. Possiamo ipotizzare che sia più vicino al gruppo "E" (concorda con questo gruppo, in casi molto particolari, cinque volte). Per quanto riguarda gli altri gruppi, concorda cinque volte con la famiglia "D" (specialmente con lo Scaphusianus 104), quattro con il gruppo "A", tre con la famiglia "P", due con le famiglie "L" e "Z" e una sola volta con i gruppi "I" e "J".

Dato che il testo della Vita, contenuto in questo codice, è molto breve e mutilo, la sua classificazione risulta impossibile. Esso contiene le seguenti varianti particolari:

\begin{tabular}{|c|c|c|c|}
\hline Parola n $^{\circ}$ & PL & Varianti & Mss. concordanti \\
\hline $47.288-289$ & $\begin{array}{c}\text { amaenissimos } \\
\text { hortulos }\end{array}$ & $\begin{array}{c}\text { amaenissimo } \\
\text { hortulo }\end{array}$ & $\begin{array}{c}23,52,80,81,110,116,116 \mathrm{a}, 119, \\
135\end{array}$ \\
\hline 48.351 & corpore & corpori & $\begin{array}{c}9,11,13,32,37,38,65,71,71 \mathrm{a}, 100, \\
110,120,140,149\end{array}$ \\
\hline $49.442-444$ & $\begin{array}{c}\text { secretiorem } \\
\text { secessit. Verum }\end{array}$ & $\begin{array}{c}\text { secreto recesser- } \\
\text { at. Uerumtamen }\end{array}$ & $\begin{array}{c}45,46,110 \\
50.478\end{array}$ \\
& revocavit & reuocauerunt & $\begin{array}{c}1,3,4,9,11,12,13,15,20,21,24, \\
58,59,66,67,80,83,84,85,100, \\
110,116 a, 127,134,138,139,142\end{array}$ \\
\hline 51.497 & praestolaretur & prestolabatur & $\begin{array}{c}11,12,20,38,58,65,83,84,100, \\
110,132\end{array}$ \\
\hline
\end{tabular}

68 A proposito del Vaticanus Ottobonianus Lat. 120, cf. Bethmann, Nachrichten, p. 358; de Meeûs, Études, p. 181; Ciccarese, Le visioni, pp. 253-273, 278-302; P. Chiesa, La traduzione latina del "Sermo in reditu reliquiarum sancti Iohannis Chrysostomi" di Cosma il Vestitore eseguita da Guarimpoto grammatico, "Aevum" 63 (1989) 158. A proposito dei codici Ottoboniani come tali, cf. ad esempio: G. Mercati, Codici latini Pico Grimani Pio e di altra biblioteca ignota del secolo XVI esistenti nell'Ottoboniana e i codici greci Pio di Modena con una digressione per la storia dei codici di S. Pietro in Vaticano, Città del Vaticano $1938=$ ST 75 (1938).

69 A proposito del Sangallensis 558, cf. Morales, Edición crítica, passim.

70 I Parisinus Lat. 3784 e Parisinus Lat. 5314 leggono: secreto secesserat. Verumtam. 


\begin{tabular}{|c|c|c|c|}
\hline Parola n $^{\circ}$ & PL & Varianti & Mss. concordanti \\
\hline \multirow{2}{*}{52.538} & \multirow{2}{*}{ quod } & quo & $5,24,73,80,81,83,94,105,106$, \\
& & leoni patri & $110,116,116 \mathrm{a}, 118,125,126,133$ \\
\hline 53.596 & Cleopatrae & \multirow{2}{*}{ leor } \\
\hline
\end{tabular}

Si può ipotizzare una vicinanza del Vaticanus Lat. 13393 al grupo "L", ma il suo testo è troppo corto e mutilo per rendere sicura la classificazione.

Per quanto riguarda gli altri codici inclassificabili, si può dire che il Vaticanus Urbinas Lat. 396 è molto vicino, primariamente, al Vaticanus Barberinianus Lat. 586, e secondariamente ai Laurentianus pl. 19, cod. 16 e Bruxellensis 64. Per quanto concerne i manoscritti classificabili, invece, esso si avvicina al gruppo "Q" (in modo particolare ai Vaticanus Barberinianus Lat. 621 e Vaticanus Urbinas Lat. 51 [107]), e alla famiglia "E" (specialmente al Sangallensis 558).

Il Basilicanus A. 4, che mostra poca propinquità con qualsiasi singolo gruppo della tradizione manoscritta della Vita (forse è più vicino ai gruppi "K" e "I"), si avvicina al Basilicanus A. 2, ossia al manoscritto che è più affine alle famiglie "K" e "R".

Infine, il codice Vindobonensis Lat. 420 ha in comune molte lezioni con i gruppi "A", "D", "E" e "L". Esso è il prodotto di tarda contaminazione alla quale fu soggetta la Vita Pauli.

Si può ipotizzare una vicinanza del Vaticanus Lat. 13393 al grupo "L", ma il suo testo è troppo corto e mutilo per rendere sicura la classificazione.

Per quanto riguarda gli altri codici inclassificabili, si può dire che il Vaticanus Urbinas Lat. 396 è molto vicino, primariamente, al Vaticanus Barberinianus Lat. 586, e secondariamente ai Laurentianus pl. 19, cod. 16 e Bruxellensis 64. Per quanto concerne i manoscritti classificabili, invece, esso si avvicina al gruppo "Q" (in modo particolare ai Vaticanus Barberinianus Lat. 621 e Vaticanus Urbinas Lat. 51 [107]), e alla famiglia "E" (specialmente al Sangallensis 558).

Il Basilicanus A. 4, che mostra poca propinquità con qualsiasi singolo gruppo della tradizione manoscritta della Vita (forse è più vicino ai gruppi "K" e "I"), si avvicina al Basilicanus A. 2, ossia al manoscritto che è più affine alle famiglie " $K$ " e " $R$ ".

Infine, il codice Vindobonensis Lat. 420 ha in comune molte lezioni con i gruppi "A", "D", "E" e "L". Esso è il prodotto di tarda contaminazione alla quale fu soggetta la Vita Pauli. 


\title{
Rękopisy Hieronimowej Vita S. Pauli Monachi Thebaei, które nie dają się sklasyfikować
}

\author{
(streszczenie)
}

Artykuł omawia te kodeksy Hieronimowej Vita S. Pauli Monachi Thebaei, które nie można jeszcze zaliczyć do żadnej z rodzin rękopiśmiennej tradycji tegoż dzieła. Już John Frank Cherf, O.S.B. uznał następujące kodeksy za niezdolne do zaliczenia do jakiejkolwiek rodziny rękopiśmiennego przekazu Hieronimowej Vita: Bernensis Lat. 111, Bruxellensis 64, Cameracensis 864 (767), Dresdensis A 62 cod. D, Laurentianus pl. 19, cod. 16, Matritensis 10007, Parisinus Lat. 3784, Parisinus Lat. 5314, Parisinus Lat. 5385, Parisinus Lat. 9741, Vaticanus Lat. 5411, Vaticanus Lat. 7014, Vaticanus Barberinianus Lat. 586, Basilicanus A. 2, Vindobonensis Lat. 420 oraz Vindobonensis S. Mariae ad Scotos 409 (324). W niniejszym studium zaś dołączyliśmy do tychże rękopisów następujące kodeksy, których Cherf nie przebadał: Iagellonicus Cracoviensis 2265 AA XII 9, Casanatensis Codex 718 (B.I.3), Lateranensis Codex A. 79, Farfensis 5 (270), Sessorianus 5 (XXIX), Vallicellanus Tomus III, Vaticanus Latinus 600, Vaticanus Lat. 5257, Vaticanus Lat. 9309, Vaticanus Lat. 13393, Vaticanus Barberinianus Lat. 469, Vaticanus Urbinas Lat. 396, Basilicanus A. 4.

Słowa kluczowe: Św. Hieronim; Vita S. Pauli Monachi Thebaei; kodykologia; paleografia

\section{Unclassified Manuscripts of St. Jerome's Vita S. Pauli Monachi Thebaei}

\author{
(summary)
}

Manuscripts which cannot be included in any of the families of the manuscript tradition of St. Jerome's Vita Sancti Pauli Primi Eremitae, examined by John Frank Cherf, O.S.B., are the following: Bernensis Lat. 111, Bruxellensis 64, Cameracensis 864 (767), Dresdensis A 62 cod. D, Laurentianus pl. 19, cod. 16, Matritensis 10007, Parisinus Lat. 3784, Parisinus Lat. 5314, Parisinus Lat. 5385, Parisinus Lat. 9741, Vaticanus Lat. 5411, Vaticanus Lat. 7014, Vaticanus Barberinianus Lat. 586, Basilicanus A. 2, Vindobonensis Lat. 420 and Vindobonensis S. Mariae ad Scotos 409 (324). To these can be added the following ones, found by the author: Iagellonicus Cracoviensis 2265 AA XII 9, Casanatensis Codex 718 (B.I.3), Lateranensis Codex A. 79, Farfensis 5 (270), Sessorianus 5 (XXIX), Vallicellanus Tomus III, Vaticanus Lat. 600, Vaticanus Lat. 5257, Vaticanus Lat. 9309, Vaticanus Lat. 13393, Vaticanus Barberinianus Lat. 469, Vaticanus Urbinas Lat. 396, Basilicanus A. 4.

Keywords: Saint Jerome; Vita S. Pauli Monachi Thebaei; Codicology; Palaeography 


\section{Bibliografia}

\section{Fonti}

Hieronymus, Vita S. Pauli Primi Eremitae, red. B. Degórski, in: Hieronymi historica et hagiographica. Vita Beati Pauli monachi Thebaei. Vita Hilarionis. Vita Malchi monachi captivi. Epistula praefatoria in Chronicis Eusebii Caesariensis. Chronicorum Eusebii Caesariensis continuatio. De viris inlustribus. In Regulae S. Pachomii versionem praefatio \| Girolamo. Opere storiche e agiografiche. Vita di san Paolo, eremita di Tebe. Vita di Ilarione. Vita di Malco, l'eremita prigioniero. Prefazione alla traduzione delle Cronache di Eusebio di Cesarea. Continuazione delle Cronache di Eusebio di Cesarea. Gli uomini illustri. Prefazione alla traduzione della Regola di Pacomio, Hieronymi opera 15, Opere di Girolamo 15, Roma 2014, 72-115.

\section{Studi}

Axters S.G., Na honderd jaar vroomheidsgeschiedenis, Gent 1973.

Barnard L.W., The date of St. Athanasius'Vita Antonii, VigCh 28 (1974) 169-175.

Baroffio B., S. Gaudenzio di Novara e S. Gaudenzio di Fiesole, „Novarien” 2 (1968) 222223.

Bartelink G., Observations de critique textuelle sur la plus ancienne version latine de la Vie de saint Antoine par saint Athanase, RBen 81 (1971) 92-95.

Bartelink G.J.M., Grécismes lexicologiques et syntaxiques dans les traductions latines du IV siècle de la "Vita Antonii” d'Athanase, „Mnemosyne” 30 (1977) 388-422.

Bartelink G.J.M., Die älteste lateinische Übersetzung der "Vita Antonii” des Athanasius im Lichte der Lesarten einiger griechischer Handschriften, „Revue d'histoire des textes" 11 (1981) 397-413.

Bethmann L., Nachrichten über die von ihm für die "Monumenta Germaniae historica" benutzten Sammlungen von Handschriften und Urkunden Italiens, aus dem Jahre 1854, „Archiv der Gesellschaft für ältere deutsche Geschichtskunde” 12 (1872) 201-426.

Bianco M.G., Gli intenti del traduttore anonimo della "Vita Antonii" di Atanasio e le sue doti letterarie, „Studi storico religiosi” 5 (1981) 223-250.

Cherf J.F., The Latin Manuscript Tradition of the "Vita Sancti Pauli", in: Studies in the Text Tradition of. St. Jerome's "Vitae Patrum”, red. W.A. Oldfather, Urbana 1943, 133-142.

Chiesa P., La traduzione latina del "Sermo in reditu reliquiarum sancti Iohannis Chrysostomi” di Cosma il Vestitore eseguita da Guarimpoto grammatico, „Aevum” 63 (1989) 147-171.

Ciccarese M.P., Le visioni di S. Fursa, „Romano Barbarica” 8 (1984-1985) 231-306.

Conte P., Osservazioni sulla leggenda di S. Cerbonio, vescovo di Populonia (H 575), „Aevum” 52 (1978) 235-260. 
Cross J.E., The "Passio s. Laurentii et aliorum": Latin Manuscripts and the "Old English Martyrology”, „Mediaeval Studies” 45 (1983) 200-213.

Cross J.E. - Tuplin C.J., An Unrecorded Variant of the "Passio S. Christinae" and the “Old English Martyrology”, ,Traditio” 36 (1980) 161-236.

Degórski B., Edizione critica della “Vita Sancti Pauli Primi Eremitae” di San Girolamo, diss. Institutum Patristicum Augustinianum, I-VI, Roma 1987 (dattiloscritto).

Degórski B., Edizione critica della "Vita Sancti Pauli Primi Eremitae” di Girolamo, Roma 1987.

Degórski B., La "conlatio codicum” della "Vita S. Pauli Primi Eremitae" (dall'inizio del primo capitolo sino alle parole: "[...] incitata sunt studia”" dello stesso capitolo), DPa 1 (1988) 57-101.

Degórski B., Un tardivo manoscritto di Cracovia contenente la "Vita Sancti Pauli Primi Eremitae” di San Girolamo, VoxP 8 (1988) 429-434.

Degórski B., La "conlatio codicum" della "Vita S. Pauli Primi Eremitae" di Girolamo, dall'inizio del II capitolo sino alle parole: "[ ...] pro Christi nomine gladio percuti" dello stesso capitolo, VoxP 9 (1989) 839-866.

Degórski B., La "conlatio codicum" della "Vita S. Pauli Primi Eremitae" di Girolamo, dall'inizio del II capitolo sino alle parole: "[ ...] pro Christi nomine gladio percuti”" dello stesso capitolo, VoxP 9 (1989) 839-866.

Degórski B., La “conlatio codicum” della "Vita S. Pauli Primi Eremitae” di san Girolamo, dalle parole: "Verum hostis callidus” del II capitolo sino alle parole: "[ ...] duo memoriae causa exempla", VoxP 10 (1990) 251-271.

Degórski B., La “conlatio codicum” della "Vita Sancti Pauli Primi Eremitae” di San Girolamo (dalle parole: "Amathas vero et Macarius" del primo capitolo sino alla fine dello stesso capitolo), RT 37 (1990) f. 4, 119-164.

Degórski B., I manoscritti delle biblioteche di Roma contenenti le traduzioni latine della Vita S. Antonii di sant'Atanasio. Alle origini della letteratura monastica, in: Cristianesimo Latino e cultura Greca sino al sec. IV. XXI Incontro di studiosi dell'antichità cristiana. Roma, 7-9 maggio 1992, Roma 1993 = SEA 42 (1993) 271303.

Degórski B., Przekaz łacińskich rękopisów Vita Sancti Pauli Primi Eremitae św. Hieronima, Katolicki Uniwersytet Lubelski. Międzywydziałowy Zakład Badań nad Antykiem Chrześcijańskim, Lublin 2000.

De Marco F., Censimento dei codici dei secoli X-XII. Roma: Biblioteca Apostolica Vaticana, Archivio di S. Pietro, SM 11 (1970) 1102-1133.

De Rossi G.B., I santi Quattro Coronati e la loro chiesa sul Celio, "Bollettino di archeologia cristiana" $3 / 4$ (1879) 45-90.

Divjak J. - Römer F., Ergänzungen zur Bibliotheca Hieronymiana manuscripta. I: Dänemark, Großbritannien, Irland, Italien, Polen, Portugal, Schweden, Spanien, Vatikan, „Scriptorium” 30 (1976) 85-113. 
Dolbeau F., Notes sur deux collections hagiographiques conservées à la Bibliothèque Vaticane, „Mélanges de l'École Française de Rome. Moyen âge - temps modernes” 87 (1975) 397-424.

Dolbeau F., Le légendier de la cathédrale de Bovino, AnBol 96 (1978) 125-152.

Dolbeau F., Un plagiat anonyme de la "Vita S. Columbani”, „Archivum Bobiense” 3 (1981) 60-63.

Dolbeau F. - Lambert P.-Y. - Lévy C. - Petitmengin P. - Tilliette J.-Y., Les Vies latines de sainte Pélagie. Inventaire des textes publiés et inédits, RechAug 12 (1977) 279-305.

Dolbeau F. - Lévy C. - Petitmengin P. - Rothschild J.-P. - Tilliette J.-Y., Les Vies latines de Sainte Pélagie. II. Compléments à l'nventaire et classement des manuscrits du texte B, RechAug 15 (1980) 265-304.

Gardner J., The Stefaneschi Altarpiece: A Reconsideration, „The Journal of the Warburg and Courtauld Institutes" 37 (1974) 58-66.

Garrison E.G., A Third “S. Bononio Manuscript” for S. Michele a Marturi, „Bibliofilia” 78 (1976) 87-105.

Gijsel J., Zu welcher Textfamilie des Pseudo-Matthäus gehört die Quelle von Hrotsvits Maria?, CM 32 (1971-1980) 279-288.

Halkin F., Sainte Tatiana: légende grecque d'une “martyre romaine”, AnBol 89 (1971) 265-309.

Huygens R.B.C., Deux sermonnaires médiévaux: Tétère de Nevers et Giraud de Barri. Textes inédits, SM 10 (1969) 271-296.

Kissane D.N., Vita Metrica Sanctae Brigidae: a critical edition with introduction, commentary and indexes, „Proceedings of the Royal Irish Academy” 77 (1977) 57-192.

Lambot C., L'homélie du Pseudo-Jérôme sur l'Assomption et l'évangile de la Nativité de Marie d'après une lettre inédite d'Hincmar, RBen 46 (1934) 265-282.

Lentini A., Il sermone di S. Bertario su S. Scolastica, „Benedictina” 1 (1947) 197-232.

Limone O., La tradizione manoscritta della "Vita Gregorii Magni” di Paolo Diacono (B.H.L. 3639). Censimento dei testimoni, SM 29 (1988) 887-953.

Massigli R., Un manuscrit inédit de l'Évangile du Pseudo-Mathieu, „Mélanges de l'École Française de Rome" 33 (1913) 81-118.

Mercati G., Opere minori raccolte in occasione del settantesimo natalizio sotto gli auspicii di S. S. Pio XI, volume I (1891-1897), Città del Vaticano 1937 = ST 76 (1937).

Mercati G., Codici latini Pico Grimani Pio e di altra biblioteca ignota del secolo XVI esistenti nell'Ottoboniana e i codici greci Pio di Modena con una digressione per la storia dei codici di S. Pietro in Vaticano, Città del Vaticano 1938 = ST 75 (1938).

Meeûs F. de, Études grégoriennes, „Scriptorium” 10 (1956) 275-281.

Morales E.M., Edición crítica de "De monacho captivo" ("Vita Malchi”) de San Jerónimo, diss. Institutum Patristicum Augustinianum, Roma 1991 (dattiloscritto).

Murjanov M., “Tatjanin den'” nella cultura russa, OCP 38 (1972) 243-252. 
Oberleitner M., Die handschriftliche Überlieferung der Werke des Heiligen Augustinus, Wien $1969=$,Sitzungsberichte des Österreichischen Akademie der Wissenschaften. Philosophisch-Historische Klasse" 263 (1969).

Oberleitner M., Die handschriftliche Überlieferung der Werke des Heiligen Augustinus, Wien $1970=$,Sitzungsberichte des Österreichischen Akademie der Wissenschaften. Philosophisch-Historische Klasse" 267 (1970).

Orselli A.M., La città altomedievale e il suo santo patrono: (ancora una volta) il "campione” pavese, „Rivista di Storia della Chiesa in Italia” 32 (1978) 1-69.

Paschini P., Ricerche agiografiche, RivAC 2 (1925) 167-194.

Philippart G., Le légendier du Schottenkloster de Wurtzbourg (Cod. Bruxellensis 64), AnBol 89 (1971) 369-375.

Poncelet A., Catalogus codicum hagiographicorum Latinorum Bibliothecarum Romanorum praeter quam Vaticanae, Bruxelles 1909.

Poncelet A., Catalogus codicum hagiographicorum Latinorum Bibliothecae Vaticanae, Bruxelles 1910.

Scicolone I., Il cardinale Giuseppe Tomasi di Lampedusa e gli inizi della scienza liturgica, „Regnum Dei” 38 (1982) 51-67.

Supino Martini P., Carolina romana e minuscola romanesca. Appunti per una storia della scrittura latina in Roma tra IX e XII secolo, SM 15 (1974) 769-793.

Supino Martini P. - Petrucci A., Materiali ed ipotesi per una storia della cultura scritta nella Roma del IX secolo, „Scrittura” 2 (1978) 55-88.

Tandoi V., Sul testo della "Vita Antonii" di Atanasio, nella più antica versione latina (note marginali a un'edizione critica recente), „Studi italiani di filologia classica” 50 (1978) 161-190.

Tomea P., L'agiografia milanese nei secoli XI e XII. Linee di tendenza e problemi. I, in: Atti dell'111 Congresso internazionale di studi sull'Alto Medioevo, Milano 26-30 ottobre 1987, Spoleto 1989, 623-688.

Verrando G.N., Liberio-Felice. Osservazioni e rettifiche di carattere storico-agiografico, „Rivista di storia della Chiesa in Italia” 35 (1981) 91-125.

Verrando G.N., La “Passio Callisti” e il santuario della Via Aurelia, „Mélanges de 1'École Française de Rome. Antiquité” 96 (1984) 1038-1083.

Weismann W., Die “Passio Genesii mimi” (BHL 3320), „Mittellateinisches Jahrbuch” 12 (1977) 22-43.

Wilmart A., Une version latine inédite de la vie de saint Antoine, RBen 31 (1914-1919) 163-173.

Wilmart A., Les rédactions latines de la vie d'Abraham ermite, RBen 50 (1938) 222-245.

Zafarana Z., Indici dei volumi I-VI, „Studi gregoriani per la storia della «libertas Ecclesiae»" 8 (1970) 1-717.

Zanetti U., Les Passions des SS. Nazaire, Gervais, Protais et Celse, AnBol 97 (1979) 6988. 